PAPER

\title{
Emotional memory and perception in temporal lobectomy patients with amygdala damage
}

\author{
B Brierley, N Medford, P Shaw, A S David
}

J Neurol Neurosurg Psychiatry 2004;75:593-599. doi: 10.1136/jnnp.2002.006403

See end of article for authors' affiliations

.....................

Correspondence to: Professor A S David, Section of Cognitive Neuropsychiatry, PO Box 68, Institute of Psychiatry and GKT School of

Medicine, De Crespigny

Park, London SE5 8AF,

UK; a.david@

iop.kcl.ac.uk

Received 2November 2002 In revised form

31 July 2003

Accepted 4 August 2003
Background: The human amygdala is implicated in the formation of emotional memories and the perception of emotional stimuli-particularly fear-across various modalities.

Objectives: To discern the extent to which these functions are related.

Methods: 28 patients who had anterior temporal lobectomy (13 left and 15 right) for intractable epilepsy were recruited. Structural magnetic resonance imaging showed that three of them had atrophy of their remaining amygdala. All participants were given tests of affect perception from facial and vocal expressions and of emotional memory, using a standard narrative test and a novel test of word recognition. The results were standardised against matched healthy controls.

Results: Performance on all emotion tasks in patients with unilateral lobectomy ranged from unimpaired to moderately impaired. Perception of emotions in faces and voices was (with exceptions) significantly positively correlated, indicating multimodal emotional processing. However, there was no correlation between the subjects' performance on tests of emotional memory and perception. Several subjects showed strong emotional memory enhancement but poor fear perception. Patients with bilateral amygdala damage had greater impairment, particularly on the narrative test of emotional memory, one showing superior fear recognition but absent memory enhancement.

Conclusions: Bilateral amygdala damage is particularly disruptive of emotional memory processes in comparison with unilateral temporal lobectomy. On a cognitive level, the pattern of results implies that perception of emotional expressions and emotional memory are supported by separate processing systems or streams.
$\mathrm{T}$ he role of the amygdala in processing emotional facial expressions, ${ }^{12}$ emotional prosody, ${ }^{34}$ and in emotional memory has been well documented. ${ }^{5-7}$ In the formation of strengthened memories for emotional, arousing material, positron emission tomography (PET) and functional magnetic resonance imaging (fMRI) studies have shown a correlation between amygdala activity in healthy volunteers watching emotional compared with neutral films or pictures, and their improved recall or recognition several days later..$^{8-10}$ Clinical studies designed to examine amygdala dependent processes have involved patients with unilateral ${ }^{1-14}$ and bilateral temporal lobe damage, ${ }^{15} 16$ and damage restricted to the amygdala as a result of Urbach-Wiethe disease ${ }^{17}$ or surgical lesions (see Adolphs et al, $1999^{18}$ for a summary). The normal enhancement of memory is lost ${ }^{5619}$ or attenuated $^{74}$ in patients with bilateral and unilateral amygdala damage, respectively.

Patients with bilateral amygdala damage appear to be specifically impaired in processing fear in facial expressions $^{118}{ }^{180}$ (but see Rapcsak et al, 2000 ${ }^{21}$ ), and in prosody. ${ }^{322}$ Neuroimaging investigations carried out in healthy volunteers $^{242324}$ are consistent with this. However, individual cases have been described with impaired recognition of fear in facial expressions which may not extend to prosody. ${ }^{18}{ }^{25}$ Furthermore, a series of temporal lobectomy patients who performed poorly in perception of emotional prosody did not overlap with those who performed poorly in tests of facial expression recognition. ${ }^{26}$

More recently, a perceptual advantage for detecting aversive stimuli under conditions of limited attention (attentional blink) has been demonstrated, which was seen to be lacking in patients with bilateral, or left amygdala, lesions. ${ }^{27}$ This has led to the hypothesis that perceptual and mnemonic functions involving emotive material are linked anatomically and functionally through the amygdala. ${ }^{27}{ }^{28}$ Recently, however, a patient with bilateral amygdala damage with impaired recognition of fear from faces and voices has been reported who showed intact affective modulation of episodic memory. ${ }^{29}$ We sought to explore this further by examining the correlation between emotional memory and affect labelling tasks in a sample of patients with varying and quantifiable lesions to the amygdala. Our aim was to contribute to a "dissection" of the cognitive or sensory components underlying emotional processing in man, with particular reference to the role of the amygdala.

We assessed 28 patients with temporal lobectomy (table 1). Eleven had left sided and 14 right sided operations; three (JC, $\mathrm{CH}$, and $\mathrm{CB}$ ) had significant and volumetrically confirmed contralateral amygdala damage on structural MRI and hence can be regarded as having bilateral damage. Patients were given standardised tests of recognition of fear, sadness, disgust, anger, and happiness in facial expressions ${ }^{3031}$ and vocal prosody. ${ }^{32}$ Affect-neutral facial perception was within the normal range ${ }^{33}$ (table 1) and matching of emotional faces and recognition of non-emotional sounds did not differ between the groups (data available on request).

The same participants were also given two tests of emotional memory: a novel test of recognition of emotional target words presented in unconnected sentences designed to tap encoding processes, and a well validated emotional story test—-the Heuer and Reisburg test. ${ }^{5635}$ All results of emotional processing tests were standardised to aid multimodal comparisons. The pattern of results on emotion

Abbreviations: NART, Nelson adult reading test; $P I Q$, performance $I Q$; $\mathrm{VIQ}$, verbal IQ; WAIS-R, Wechsler adult intelligence scale, revised 
Table 1 Detailed profiles of temporal lobe epilepsy patients: demographic, clinical, and neuropsychological variables

\begin{tabular}{llll}
\hline & Left TL & Right TL & Bilateral amygdala \\
lesions
\end{tabular}

recognition and emotional memory tests was compared across and within subjects.

\section{METHODS \\ Participants}

Patients had all been treated for otherwise intractable epilepsy at King's College Hospital, London, with en-bloc resection of the anterior temporal lobe. ${ }^{36}$ Mean age was 37.4 years, and estimated IQ from the Nelson adult reading test $(\mathrm{NART})^{37}$ was 100.2 (range 77 to 127 ) (details in table 1 ). Matched healthy controls were also tested for all comparisons alongside the patients over the course of a year. The controls were drawn from a pool of 40 local volunteers free of neurological and psychiatric illness who had made themselves available to assist with research, with 20 or more acting as controls for each test. Their mean age was 34.2 years (range 19 to 67 ), and their NART IQ was 104.4 (range 75 to 127). Handedness was assessed using the Annett scale. ${ }^{38}$ Four patients were not right handed - two with left sided and two with right sided lobectomies. The main statistical analyses were group comparisons with 32 normal volunteers for the expression tests and 20 for the memory tests. Subjects were not individually matched, yet in all experiments there were no differences approaching significance between patient and control groups in terms of age, sex, or IQ.

\section{Magnetic resonance imaging}

Structural MR images were acquired using a $1.5 \mathrm{~T}$ GE N/Vi Signa System (General Electric, Milwaukee, Wisconsin, USA). A three dimensional, inversion recovery prepared, fast spoiled GRASS Tl weighted dataset was obtained in the coronal plane with $1.5 \mathrm{~mm}$ contiguous sections. Amygdala volume (in $\mathrm{mm}^{3}$ ) was defined according to modified Watson criteria. ${ }^{39} 40$ "Bilateral patients" were operationally defined as unilateral resection (no amygdala or remnant volume $>4$ SD below controls mean) plus contralateral amygdala volume reduction of $\geqslant 1.5$ SD below the mean of 32 age matched volunteers (mean (SD), 2404.8 (360.0) $\mathrm{mm}^{3}$ ). Contralateral amygdala volumes in the patients with bilateral lesions were, for $\mathrm{CB}, 1.5 \mathrm{SD}$ below control mean, for JC, $>2 \mathrm{SD}$, and for $\mathrm{CH},>5$ SD (fig 1, A-D).

\section{Stimuli}

\section{Expressions}

We used the "megamix" test of expression recognition of morphed, interpolated expressions of fear, disgust, anger, sadness, and happiness taken from the Ekman and Friesen series, ${ }^{20}{ }^{32}$ and a test of prosody with a speaker counting in the tone of voice corresponding to each of the same emotions. ${ }^{3}{ }^{4}$ Subjects chose the most appropriate label for each from the list of possible labels. Subjects were required to make a response, even if unsure. The proportion of correct responses was used in the analysis. Mean scores were used in the analysis. The distribution of scores showed some positive skew but was sufficiently normal to permit the use of parametric statistical tests.

\section{Emotional memory}

We used the Heuer and Reisburg test (1990) developed by Cahill and others, ${ }^{56814}$ and a novel test of matched emotional and neutral, unconnected sentences (available on request). The novel test comprises 42 unconnected sentences read by the subject. Forced choice recognition of emotional and neutral "target" words was tested one hour after presentation of the stimuli. ${ }^{41}$ Emotional target words were selected from the affective norms for English words $(\mathrm{ANEW})^{42}$ - a list of over 1000 words each rated for valence, arousal, dominance, and frequency. Emotionally aversive words were selected with a high score for "arousal" (6-9 from a maximum score of 9), and a low score (1-3; that is, sad) for "valence." These words were labelled the "emotional target" words. The sentences were designed to be short and simple, yet interesting and provocative, and described basic events or situations-for example, "The sailor was responsible for the rape; he would abuse the children at every party."

Matched "neutral target" words were generated by submitting each "emotional target" word independently to a validated psycholinguistic database. ${ }^{43}$ The lists of words so produced were by specific request the same length as each emotional target word, and matched to within I SD for frequency, ${ }^{44}$ imagability, concreteness, and familiarity (see http://wapsy.psy.uwa.edu.au/mrc2.html). One "neutral target" word (neutral in tone) was selected from this sublist which maintained the sense of the sentence, to replace the emotional target word; for example: "The sailor was responsible for the dock; he would amuse the children at every party." This created a set of neutral sentences well matched to the emotional sentences.

Memory for all target words was tested by presenting each with two distracters giving a one-in-three forced choice recognition test, to avoid ceiling effects. The distracters were 


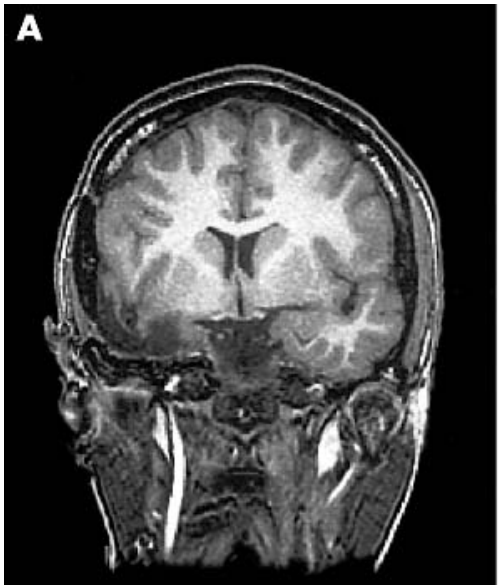

$\mathrm{R}$

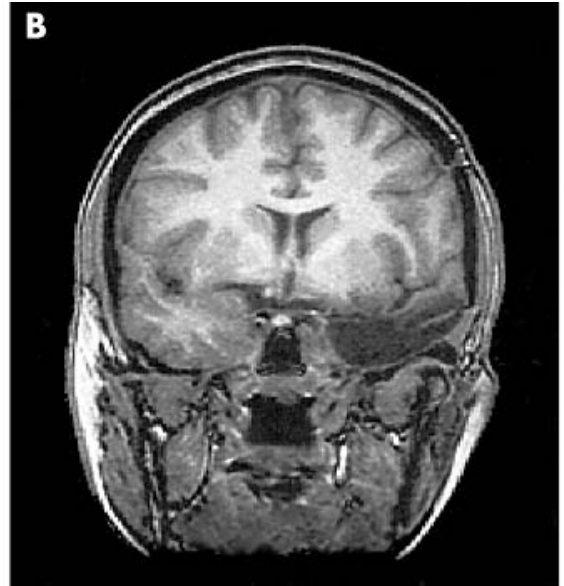

L R

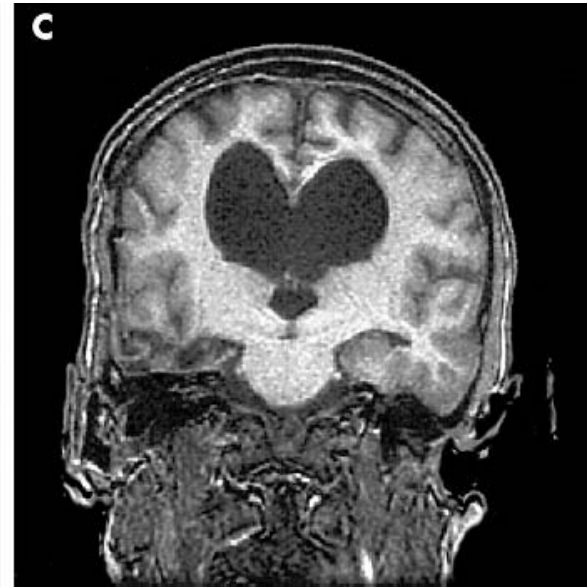

L R

Figure 1 Coronal section magnetic resonance images (MRI) of the three patients with bilateral amygdala damage. (A) Patient $C B$, right temporal lobectomy with contralateral amygdala damage: 22 year old woman with temporal lobectomy at age 19. Seizure-free and drug-free since the operation. Full scale $I Q=85$; NART estimated $I Q=85$. (B) Patient JC, left temporal lobectomy with contralateral amygdala damage: 27 year old woman with temporal lobectomy at age 25. Epilepsy started at age 12. Preoperative MRI showed a low grade tumour of the left amygdala and anterior hippocampus. The right amygdala and hippocampal tissue was also found to be sclerotic. Full scale IQ=76; NART estimated $I Q=77$. (C) Patient $C H$, right temporal lobectomy with contralateral amygdala damage and dilated lateral and third ventricles; 35 year old man with temporal lobectomy at age 25. Preoperative MRI consistent with encephalitis. Epilepsy at age 2. Seizure-free since surgery. Full scale $I Q=86$; NART estimated $I Q=97$. WMS scores largely in line with $I Q$. (D) Tissue volumes of the three bilateral cases as a proportion of the control mean. NART, Nelson adult reading test; WMS, Wechsler memory scale.

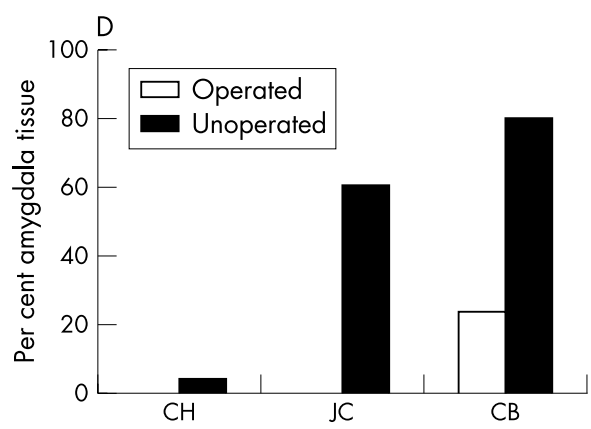

selected from the same database list from which the matched neutral target word was chosen, so the distracters were also matched (to within I SD) to the emotional target word for frequency, imagability, concreteness, and familiarity, as well as arousal and valence-for example, porn/rape/hook, and seam/dock/curb. An emotional "target index," representing the degree of emotional enhancement in retrieval, was calculated as a proportion of correct recognition scores for the emotional target words minus neutral targets over combined neutral and emotional target word recognition.

The modified Heuer and Reisburg test of emotional memory comprises 11 picture slides and accompanying text, depicting a story of a boy who suffers a terrible car accident, after which he is rushed to theatre in hospital. This emotional, central section of the story follows and precedes neutral sections that set the scene and describe the repercussions, respectively. Memory for story details is tested one week after presentation with a multiple choice questionnaire. Retrieval of information relevant to emotional and neutral pictures is compared, and the data used to calculate an emotional index score as above.

\section{RESULTS}

\section{Expression recognition}

Analysis of variance (ANOVA) was used to compare the performance of patients with left and right sided temporal lobectomy (within-group contrast) in recognising expressions of fear, anger, sadness, disgust, and happiness in the facial expression (megamix) and prosody tests against normal controls. There was no effect of side of lobectomy $(\mathrm{F}(1,279)=2.42, \mathrm{p}=0.12)$, but there was a significant effect of test $(\mathrm{F}(1,279)=5.30, \mathrm{p}=0.02)$ and emotion $(\mathrm{F}(4,279)=5.28, \mathrm{p}<0.001)$. Post hoc Tukey tests showed that recognition of expressions of fear and anger was inferior to recognition of sadness $(\mathrm{p}=0.03$ and $\mathrm{p}<0.001$, respectively). Anger recognition was also worse than recognition of disgust $(\mathrm{p}=0.01)$. There were no significant interactions between side and emotion $(\mathrm{F}(4,279)=1.03, \mathrm{p}=0.39)$, or side and test $(F(1,279)=1.75, p=0.19)$, and there was no three way interaction between test, side, and emotion $(\mathrm{F}(4,279)=0.53, \mathrm{p}=0.71)$. However, emotion and test interacted significantly $(\mathrm{F}(4,279)=2.71, \mathrm{p}=0.03)$-that is, recognition of different emotions depended on the modality of the stimuli (see table 2 ).

Table 2 shows the results of simple contrasts ( $t$ tests) between the temporal lobe group and the normal comparison group on recognition of emotional expressions: 32 participants for faces and 29 of these for voices. Percentage correct for each emotion was the dependent variable. Both visual and auditory tests revealed significant deficits in the recognition of fear and anger. Pearson correlations were carried out on $z$ scores for each emotion, calculated on performance for the megamix faces against prosody tests for the unilateral lobectomy groups against healthy volunteers. All were significant except happiness $(r=0.09)$. They ranged from 0.37 (sad), to 0.39 (disgust), 0.59 (fear), and 0.68 (anger).

\section{Cases with bilateral amygdala damage}

Performance on the prosody and megamix tests of emotion recognition was compared with healthy controls for each of three temporal lobectomy patients with bilateral amygdala damage. Patient CB was the least impaired overall, performing as well as, or better than, controls for all tests except fear prosody, which was marginally worse than normal, while recognition of facial expressions of fear was superior. JC showed a similar pattern of performance in both sensory 
Table 2 Emotion recognition from faces and prosody responses: patients with unilateral and bilateral amygdala damage

\begin{tabular}{|c|c|c|c|c|c|c|}
\hline \multirow[b]{2}{*}{ Emotion } & \multicolumn{6}{|c|}{$\%$ Correct } \\
\hline & $\mathrm{JC}$ & $\mathrm{CH}$ & CB & \multicolumn{2}{|c|}{ Unilateral TL $(n=25)$} & Control $(n=32)$ \\
\hline \multicolumn{7}{|l|}{ Faces } \\
\hline Happy & 100 & $65^{* *}$ & 95 & \multicolumn{2}{|l|}{$84.7(21.0)$} & $91.1(12.5)$ \\
\hline Fear & 50 & $15^{*} \dagger$ & 95 & \multicolumn{2}{|c|}{51.2 (28.0)‡ } & $65.2(25.8)$ \\
\hline Sad & 90 & $35^{*} \dagger$ & 80 & \multicolumn{2}{|c|}{$69.1(28.6)$} & $79.7(22.7)$ \\
\hline Disgust & $45^{*}$ & $50^{*}$ & 90 & \multicolumn{2}{|c|}{$67.0(26.8) \ddagger$} & $79.1(21.5)$ \\
\hline Anger & $55^{*}$ & $15^{* \star} \dagger$ & 85 & \multicolumn{2}{|c|}{$56.6(28.1) \ddagger$} & $81.6(17.7)$ \\
\hline Voices & & & & \multirow{2}{*}{\multicolumn{2}{|c|}{$56111761+$}} & $(n=29)$ \\
\hline Happy & 100 & 80 & 80 & & & 73.7 (16.7) \\
\hline Fear & $30 * *$ & $50^{*}$ & 70 & \multicolumn{2}{|c|}{$54.8(24.9)$} & $71.7(20.0)$ \\
\hline Sad & 100 & 80 & 90 & \multicolumn{2}{|l|}{$78.4(15.1)$} & $75.0(22.6)$ \\
\hline Disgust & 30 & 30 & 80 & \multicolumn{2}{|l|}{$44.8(23.5)$} & $48.7(25.4)$ \\
\hline \multirow[t]{2}{*}{ Anger } & 90 & $30^{*} \dagger$ & 70 & \multicolumn{2}{|l|}{$56.5(20.9)$} & $69.7(20.4)$ \\
\hline & \multicolumn{6}{|c|}{ Emotional index } \\
\hline Memory & $\mathrm{JC}$ & $\mathrm{CH}$ & CB & \multicolumn{3}{|c|}{ Left TL $(n=11)$ Right TL $(n=14)$ Control $(n=20)$} \\
\hline \multicolumn{7}{|c|}{ Heuer and Reisberg $\S:$} \\
\hline Emotional & 50 & 23 & 20 & 42.8 & 44.5 & 56.1 \\
\hline Neutral & 54.5 & 39.5 & 27 & 36.9 & 34.0 & 48.2 \\
\hline Index (\%) & $-4.3^{*}$ & $-26.4^{\star *}$ & $-14.9^{* *}$ & $7.4(27.3)$ & 12.8 (14.3) & $7.6(9.7)$ \\
\hline \multicolumn{7}{|l|}{ Sentences $\approx$ : } \\
\hline Emotional & 51.1 & 52.4 & 76.2 & 66 (18) & 71 (17) & 79 (14) \\
\hline Neutral & 38.1 & 28.6 & 76.2 & 54.5 (14) & 59 (15) & 64 (13) \\
\hline Index (\%) & 20.0 & $-29.4^{\star *}$ & 0.0 & $9.6(12.4)$ & $9.3(10.9)$ & 10.5 (13.3) \\
\hline \multicolumn{7}{|c|}{$\begin{array}{l}\text { Values are raw scores or mean (SD). } \\
{ }^{*} 1 \text { SD less than healthy control; }{ }^{* *} 2 \text { SD less than healthy control. } \\
\dagger 1 \text { SD less than unilateral temporal lobectomy. } \\
\text { †Temporal lobectomy patients significantly less than healthy controls ( } t \text { test, } p<0.05 \text { ). } \\
\text { §There are } 11 \text { slides, each with between } 6-9 \text { questions. Each question is a } 1 \text { in } 4 \text { multiple choice. Chance }=25 \% \text {. } \\
\text { TRecognition memory scores (three-alternative, forced choice) of } 21 \text { emotional, } 21 \text { neutral, given as per cent. } \\
\text { Chance }=33 \% \text {. } \\
\mathrm{JC} / \mathrm{CH} / \mathrm{CB} \text {, initials of patients with bilateral amygdala damage; TL, temporal lobectomy. }\end{array}$} \\
\hline
\end{tabular}

modes for all emotions except anger: she demonstrated superior performance in recognising anger prosody, but clear deficits in recognising anger in facial expressions. Patient $\mathrm{CH}$ performed poorly overall, although he was worse in his recognition of all emotions in facial expressions than in prosody: recognition of happiness and sadness in prosody were in the normal range (table 2).

\section{Emotional memory}

The effect of emotional memory was examined by contrasting performance on the neutral and emotional stimuli, within each of the two tests, and within the temporal lobectomy and control group separately, using paired $t$ tests. A significant superiority in performance with emotional material was seen in both groups on both tests (all $\mathrm{p}<0.01$ ). However, when examined separately, the left lobectomy group just failed to show significant enhancement on the Heuer and Reisberg test $(\mathrm{p}=0.12)($ table 2$)$.

Emotional index scores were calculated from the two emotional memory tests and converted into $z$ scores using the means and standard deviations from the 20 matched healthy controls. The emotional sentences index and the index calculated for the Heuer and Reisburg test showed no significant correlation in either left (Pearson: $r=0.32$, $\mathrm{p}=0.31)$, or right temporal lobectomy patients $(r=0.43$, $\mathrm{p}=0.14)$, but a weak positive association when all patients with temporal lobectomy were combined $(r=0.33, \mathrm{p}=0.09)$. Separate ANOVAs contrasting unilateral cases with 20 normal controls (drawn from the same pool as those who participated in the expression studies) were carried out on the two tests, with emotional index as the dependent variable in each. The results did not approach significance and this held true whether the unilaterals were combined or whether right and left sided groups were analysed separately.

Pearson correlations were determined between each of the emotions tested in the facial and prosody tests, and the two emotional memory indices for all the unilateral lobectomy patients given the four tests. Prosody scores did not correlate significantly with either of the emotional memory indices, for any emotion. The emotional indices generated from the Heuer and Reisburg test also did not correlate significantly (all $\mathrm{p}>0.2$ ) with expression recognition megamix scores (see fig 2). The correlation for fearful faces was $r=0.2, \mathrm{p}=0.2$.

Megamix scores for expressions of fear $(r=0.35, \mathrm{p}=0.07)$ sadness $(r=0.54, \mathrm{p}=0.003)$, and happiness $(r=0.40$, $\mathrm{p}=0.03$ ) were, however, observed to correlate significantly with the emotional memory index from the sentences test.

Figure 3 shows the pattern when performance is plotted for each of the four tasks (two affect recognition and two emotional memory), concentrating on fear recognition from the faces and prosody tests. All patients with temporal lobectomy performed somewhat worse than healthy controls overall $(<1$ SD below the mean) in facial and prosody expression recognition. Both fig 2 and fig 3 show that patients with unilateral damage performed reasonably well on the Heuer and Reisburg test of emotional memory (the left temporal lobectomy group non-significantly worse than the right). However, the bilateral patients' emotional sentences index scores deviated significantly from unilateral patients' scores, and this was accentuated for the emotional index calculated from the Heuer and Reisburg illustrated story test.

When emotional memory in patients with bilateral amygdala damage was examined separately (within subject), $\mathrm{CH}$ and $\mathrm{CB}$ performed more poorly on both tests of emotional 


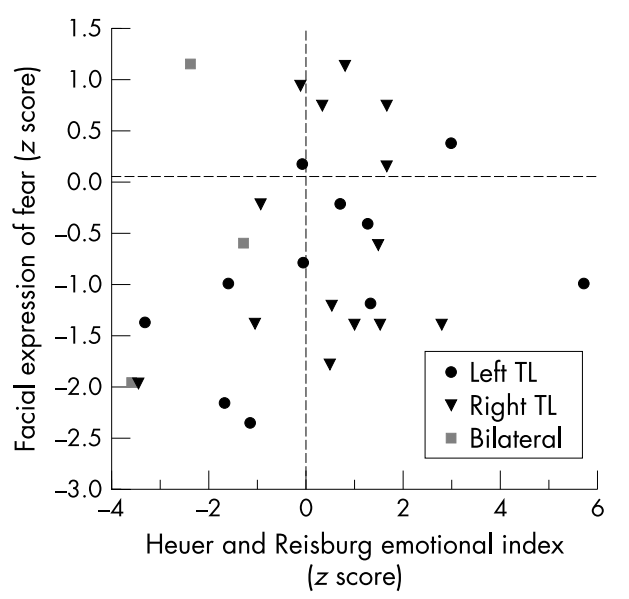

Figure 2 Correlation between fear recognition performance and emotional memory enhancement. Cases in the upper left and lower right quadrant represent impaired performance on one task alongside intact or superior performance on the other-evidence of a dissociation of functions. TL, temporal lobe.

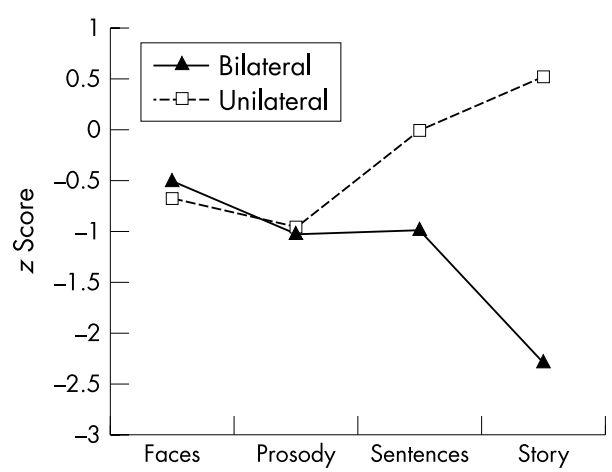

Figure 3 Temporal lobectomy patients with unilateral and bilateral amygdala damage: performance on all tasks for fear.

memory compared with all other tests of emotional processing. CB was unusual in showing well preserved fear recognition but no emotional memory enhancement (her performance on the Heuer and Reisberg test was generally poor while that on the sentences was good). JC showed more enhancement on the emotional sentences than controls, although she too showed poor emotional enhancement for the Heuer and Reisburg test.

\section{DISCUSSION}

This study is the first to assess systematically and in a standardised fashion, the relation between emotional memory and perception in a group of patients with temporal lobectomy who had varying degrees of amygdala damage and a wide range of abilities. The main finding is a lack of association between measures of emotional memory enhancement and recognition of expressions of fear. This tends to undermine the view that both functions reflect a single underlying cognitive process, ${ }^{27-29}$ though it remains possible that they both normally require the integrity of the amygdala.

Our results for the recognition of emotions in the visual and auditory modalities among subjects with unilateral temporal lobectomies are broadly in line with previous findings. ${ }^{45-47}$ There was general impairment in the recognition of emotions with negative valence, particularly those that have been linked with social approach or avoidance behaviours. The poor correlation between happiness recognition in voices and faces could be interpreted as evidence for modality specificity, but it may simply reflect psychometric factors, as expressions of happiness are strikingly easy to recognise from faces but much less so from prosody, judging by the controls' mean scores.

The bilaterally affected subjects as a group showed overall deficits in emotional recognition across modalities. Considering the categorical perception of fear stimuli, two of the three bilateral patients showed recognition deficits across modalities but it was notable that one (CB) performed in the upper half of the normative range. Thus, while the intact amygdala may usually be required for correct naming of emotional expressions of fear, performance in this sphere may be achieved despite considerable damage to the structure. CB showed damage similar in degree to that of other reported case studies of bilateral amygdala damage with significantly impaired fear recognition, ${ }^{20}$ so this heterogeneity is unlikely to be due simply to the extent of the amygdala lesions. Instead it may involve the presence or absence of damage to specific nuclei or pathways.

Another bilateral patient, JC, showed very poor recognition of anger in facial expressions, but appeared to be superior to controls in recognising anger in prosody. This dissociation indicates that different processes and perhaps neural systems-including the orbito-frontal cortex as well as the amygdala-underlie the recognition of anger across the two modalities. $^{48}$

The findings for the tests of emotional memory show a notable divergence between the effects of unilateral and bilateral damage. The performance of subjects with unilateral lesions was comparable to that of matched healthy controls on both measures of emotional memory. There was a suggestion of an effect of laterality on emotional memory that was less evident in the left lobectomy group, as shown previously in behavioural studies ${ }^{14}$ but in contrast to some functional imaging studies. ${ }^{8}{ }^{10}$ The ability to form enhanced emotional memories was seriously compromised when the damage to the amygdala was bilateral-in line with previous work. $^{5-7}$ Excepting the normal pattern of performance of JC on the sentences test, the overall picture is that the faces and prosody tests appear to be less sensitive tests of bilateral damage than those of emotional memory.

Our assessment of emotional memory was designed to tap both encoding (with the novel sentences test) and consolidation (with the Heuer and Reisburg test). The positive association we report between the two tests of emotional memory could be taken as lending support to the connection proposed by Cahill et al between long term consolidation and early/immediate changes at encoding. ${ }^{8}$ Functional MRI has revealed amygdala activation during encoding, which correlates both with immediate and delayed (four weeks) recall of pleasant and unpleasant stimuli in healthy populations. ${ }^{9}{ }^{28}$ However, the positive association between the two different indices of emotional memory we used is weak, with a shared variance of around $10 \%$. Therefore, while the mechanism which underlies short term emotional memory formation may be related to long term memory formation, these two aspects of memory are in no way synonymous. Another explanation for the fMRI results is that activation of the amygdala complex seen during emotional perceptual tasks may reflect activity within a much wider network, which includes visual processing and attentional systems involving the occipital, temporal, and medial frontal cortices. ${ }^{28} 4950$

The most striking overall finding was the lack of an association between the results of the perceptual and mnemonic tests of emotional processing. For the bilateral and unilateral subjects no significant correlation was observed between the Heuer and Reisburg test and the 
measures of emotion perception. This lack of association was also evident in individual cases: $\mathrm{CB}$, who has bilateral amygdala damage, showed superior fear perception but a complete absence of emotional memory enhancement. Several subjects showed normal or superior emotional enhancement of memory but impairment of up to 2 SD below the control mean on fear recognition, the same combination shown in patient DR recently reported by Papps and colleagues. ${ }^{29}$ Together, these cases imply the presence of a double dissociation.

The pattern was somewhat different on the novel test of emotional memory. In the unilateral subjects, performance on expression recognition scores of fear, sadness, and happiness correlated with that on the sentences test of emotional memory. This relation might be taken to reflect similarities in the perception and encoding of the two sorts of emotional stimuli. Similarly, healthy controls but not subjects with bilateral amygdala damage have previously been shown to have a perceptual ${ }^{27}$ as well as recall ${ }^{11}{ }_{12}$ advantage for verbal emotional stimuli. However, such a conclusion is undermined by consideration of the results from the subjects with quantified bilateral damage (figs 2 and 3). There is again some divergence between the recognition of emotion in facial expressions and the formation of emotional memory assessed by our novel test, indicating that different processes might underlie performance on these tasks.

Another explanation for this pattern of results is that connections from, say, the fusiform gyrus to the amygdala may be disrupted, resulting in problems of identifying facial expressions alone, while damage to connections from, for example, the hippocampus might impair emotional memory, leaving processing of expressions intact. Hence apparent dissociations could occur despite both functions relying on a common processing mechanism in the amygdala (we thank an anonymous referee for this suggestion). Distinguishing between the "connectionist" and multiple processor accounts of such dissociations may be possible anatomically if not behaviourally.

It must be acknowledged that all the patients in the current study had brain damage from their underlying condition plus surgery which extended well beyond the amygdala, including the temporal pole and the hippocampus. Hence, attributing performance entirely to the presence or absence of a healthy amygdala cannot be sustained. Were there to be widespread cognitive deficits, this might be attributed to such extra-amygdala damage. Recall and recognition on the specific memory tests were poorer in the patients than in the controls. Indeed most of the cases showed some generalised deficits. However, as such deficits tend to be minimal this issue is less relevant and does not detract from the overall pattern of preserved and impaired abilities. Finally, the lack of significant association on some of the contrasts between cases and comparison groups may have reflected inadequate statistical power.

We interpret the general pattern of performance recorded here as suggesting specific and distinct roles for the amygdala (and its connections) in emotional expression recognition and emotional memory formation. Case SP, who has UrbachWiethe disease, has more uniform and complete amygdala degeneration and shows severe impairment of fear perception and emotional memory. However, the presence of such an association does not rule out the more theoretically decisive dissociation of functions. ${ }^{50}$ Perhaps the dissociations we and others report are more likely to occur where damage to the amygdala- a collection of nuclei with distinct projectionsarises through surgical intervention or through the less predictable effects of mesial temporal sclerosis. The basolateral nuclei in particular have been linked to memory modulation from animal studies, ${ }^{51}$ although there are extensive direct and indirect projections from visual and other unisensory and polysensory receptive cortices to the lateral nucleus. ${ }^{53}$ Neuroimaging with spatial resolution at the level of nuclear groups in the amygdala is needed before specific brain-behaviour correspondence can be achieved in living humans. In the meantime this study shows that the processes of perceiving and remembering emotional material may dissociate at the behavioural level.

\section{ACKNOWLEDGEMENTS}

We thank Charles Polkey, Robin Morris, Larry Cahill, Andy Calder, and clinicians at the Regional Neurosciences Centre, King's College Hospital, London, for their help with various aspects of the study, which was supported by the Fund for Epilepsy and Remedi.

\section{Authors' affiliations}

B Brierley, N Medford, P Shaw, A S David, Section of Cognitive Neuropsychiatry, Institute of Psychiatry and GKT School of Medicine, De Crespigny Park, London, UK

Competing interests: none declared

\section{REFERENCES}

1 Adolphs R, Tranel D, Damasio $H$, et al. Impaired recognition of emotion in facial expressions following bilateral damage to the human amygdala. Nature 1994:372:669-72.

2 Morris JS, Frith C, Perrett DI, et al. A differential neural response in the human amygdala to fearful and happy facial expressions. Nature 1996;383:812-15

3 Scott SK, Young AW, Calder AJ, et al. Impaired auditory recognition of fear and anger following bilateral amygdala lesions. Nature 1997;385:254-7.

4 Phillips ML, Young AW, Scot SK, et al. Neural responses to facial and vocal expressions of fear and disgust. Proc $R$ Soc Lond 1998;265:1809-17.

5 Cahill L, Babinsky R, Markowitsch HJ, et al. The amygdala and emotional memory. Nature 1995;377:295-6.

6 Adolphs R, Cahill L, Schul R, et al. Impaired declarative memory for emotional material following bilateral amygdala damage in humans. Learning Memory 1997:4:291-300.

7 Phelps E, LaBar K, Anderson A, et al. Specifying the contributions of the human amygdala to emotional memory: a case study. Neurocase 1998;4:527-40.

8 Cahill L, Haier RJ, Fallon J, et al. Amygdala activity at encoding correlated with long-term, free recall of emotional information. Proc Natl Acad Sci USA 1996;93:8016-21.

9 Hamann SB, Ely TD, Grafton ST, et al. Amygdala activity related to enhanced memory for peasant and aversive stimuli. Nat Neurosci 1999;2:289-93.

10 Canli T, Zhao Z, Brewer J, et al. Event-related activation in the human amygdala associates with later memory for individual emotional experience. J Neurosci 2000;20:1-5.

11 Phelps E, LaBar K, Spencer D. Memory for emotional words following unilateral temporal lobectomy. Brain Cogn 1997;35:85-109.

12 LaBar KS, Phelps EA. Arousal-mediated memory consolidation: role of medial temporal lobe in humans. Psychol Sci 1998;9:490-3.

13 Anderson A, Spencer DD, Fulbright RK, et al. Contribution of the anteromedial temporal lobes to the evaluation of facial emotion. Neuropsychology 2000; 14:526-36.

14 Adolphs R, Tranel D, Denburg N. Impaired emotional declarative memory following unilateral amygdala damage. Learning Memory 2000;7:180-6.

15 Schmolck H, Squire LR. Impaired perception of facial emotions following bilateral damage to the anterior temporal lobe. Neuropsychology 2001;15:30-8.

16 Broks P, Young AW, Maratos EJ, et al. Face processing impairments after encephalitis: amygdala damage and recognition of fear. Neuropsychologia 1998;36:59-70

17 Babinsky R, Calabrese P, Durwen HF, et al. The possible contribution of the amygdala to memory. Behav Neurol 1993;6:167-70.

18 Adolphs R, Tranel D, Hamann S, et al. Recognition of facial emotion in nine individuals with bilateral amygdala damage. Neuropsychologia 1999;37:1111-17

19 Mori E, Ikeda M, Hirono N, et al. Amygdala volume and emotional memory in Alzheimer's disease. Am J Psychiatry 1999;156:216-22.

20 Calder AJ, Young AW, Rowland D, et al. Facial emotion recognition after bilateral amygdala damage: differentially severe impairment of fear. Cogn Neuropsychol 1996;13:699-745.

21 Rapcsak SZ, Galper SR, Comer JF, et al. Fear recognition deficits after focal brain damage. A cautionary note. Neurology 2000;54:575-81.

22 Adolphs R, Tranel D. Intact recognition of emotional prosody following amygdala damage. Neuropsychologia 1999;37:1285-92.

23 Sprengelmeyer R, Young AW, Schroeder U, et al. Knowing no fear. Proc R Soc Lond 1999;266:2451-6. 
24 Dolan RJ. Crossmodal binding of fear in voice and face. Proc Natl Acad Sci USA 2001;98:10006-10.

25 Anderson AK, Phelps E. Intact recognition of vocal expressions of fear following bilateral lesions of the human amygdala. Neuroreport 1998:9:3607-13

26 Adolphs R, Tranel D, Damasio $\mathrm{H}$. Emotion recognition from faces and prosody following temporal lobectomy. Neuropsychology 2001;15:396-404. 27 Anderson AK, Phelps EA. Lesions of the human amygdala impair enhanced perception of emotionally salient events. Nature 2001;411:305-9.

28 Hamann SB. Cognitive and neural mechanisms of emotional memory. Trends Cogn Sci 2001;5:394-400.

29 Papps BP, Calder AJ, Young AW, et al. Dissociation of affective modulation of recollective and perceptual experience following amygdala damage. J Neurol Neurosurg Psychiatry 2003;74:253-4.

30 Ekman P, Friesen WV. Pictures of facial affect. Palo Alto: Consulting Psychologists Press, 1976.

31 Young AW, Rowland D, Calder A, et al. Facial expression megamix: tests of dimensional and category accounts of emotion recognition. Cognition 1997:63:271-313

32 Calder AJ, Keane J, Manes F, et al. Impaired recognition and experience of disgust following brain injury. Nat Neurosci 2000;3:1077-8.

33 Benton AL, Sivan AB, Hamsher KdeS, et al. Contributions to neuropsychological assessment: a clinical manual, 2nd ed. New York: Oxford University Press, 1994.

34 Maratos EJ, Dolan RJ, Morris JS, et al. Neural activity associated with episodic memory for emotional context. Neuropsychologia 2002;39:910-20.

35 Hever F, Reisburg D. Vivid memories of emotional events: the accuracy of remembered minutiae. Memory Cogn 1990;18:496-506.

36 Polkey CE. Anterior temporal lobectomy at the Maudsley Hospital, London. In: Engel J, ed. Surgical treatment of the epilepsies. New York: Raven Press, 1987:641-5.

37 Nelson $\mathrm{H}$, Willison J. National adult reading test manual (NART). Windsor: NFER-Nelson, 1991.

38 Annett $M$. The binomial distribution of right, mixed and left handedness. Q J Exp Psychol 1967; 19:327-33.
39 Watson C, Andermann F, Gloor P, et al. Anatomic basis of amygdaloid and hippocampal volume measurement by magnetic resonance imaging Neurology 1992;42:1743-50

40 Brierley B, Shaw $P$, David AS. The human amygdala: a systematic review and meta-analysis of volumetric MRI. Brain Res Rev 2002:39:84-105.

41 Kensinger EA, Brierley B, Medford N, et al. Effects of normal aging and Alzheimer's disease on emotional memory. Emotion 2002;2:118-34.

42 Bradley MM, Lang PJ. Affective norms for English words (ANEW). The NIMH Center for the Study of Emotion and Attention. Miami: University of Florida, 1999

43 Coltheart M. The MRC psycholinguistic database. Q J Exp Psychol 1981;33A:497-505.

44 Kucera H, Francis W. Computational analysis of present-day American English. Providence: Brown University Press, 1967.

45 Borod JC, Cicero BA, Obler LK, et al. Right hemisphere emotional perception: evidence across multiple channels. Neuropsychology 1998; 12:446-458

46 Calder AJ, Lawrence AD, Young AW. Neuropsychology of fear and loathing. Nat Rev Neurosci 2001;2:352-63.

47 Kucharska-Pietura K, Phillips ML, Gernand W, et al. Perception of emotions from faces and voices following unilateral brain damage. Neuropsychologia 2003;41:1082-90

48 Blair RJ, Morris JS, Frith CD, et al. Dissociable neural responses to facial expressions of sadness and anger. Brain 1999;122:883-93.

49 Adolphs R. Neural systems for recognizing emotion. Curr Opin Neurobiol 2002;12:169-77.

50 Shallice T. From neuropsychology to mental structure. Cambridge: Cambridge University Press, 1988.

51 McGaugh JL, Ferry B, Vazdarjanova A, et al. Amygdala: role in modulation of memory storage. In: Aggleton JP, ed. The amygdala, 2nd ed. Oxford: Oxford University Press, 2002:391-424.

52 Aggleton JP, Saunders RC. The amygdale - what's happened in the last decade. In: Aggleton JP, ed. The amygdala, 2nd ed. Oxford: Oxford University Press, 2002:2-30.

53 Warrington EK. The Camden memory tests. Brighton: Psychology Press, 1996

\section{$\mathrm{ECHO}$}

\section{Intravenous immunoglobulins may have role in vasculitic neuropathy}

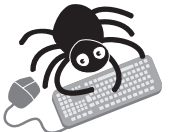

Please visit the Journal of Neurology, Neurosurgery, and Psychiatry website [www. innp.com] for a ink to the full text of this article. ntravenous immunoglobulins may be beneficial in vasculitic peripheral neuropathy, especially in acute multisystem diseases such as systemic lupus erythematosus and Sjögren's syndrome.

Treatment with intravenous immunoglobulins may be considered as the sole or as adjuvant treatment, particularly in patients in whom other immunosuppressive treatment has failed.

A study from Israel followed six patients with various inflammatory diseases accompanied by vasculitic peripheral neurophysiological that were unresponsive to conventional immunosuppressive treatment. Two patients had an acute peripheral neuropathy and four a chronic one.

All patients received the regular high intravenous dose of immunoglobulin and the investigators followed their progress by noting subjective clinical improvement, and serological neurophysiological investigations.

Four patients responded to the treatment, including the two patients with acute neuropathies. The patients with sarcoidosis and mixed cryoglobulinaemia did not respond.

The investigators think that the beneficial effects of intravenous immunoglobulin in the four patients are multifactorial, acting through complement deactivation, receptor blockade, anti-idiotypes, and modulation of cytokine production.

Patients were more likely to achieve remission when treated during an acute or subacute phase, rather than after longstanding disease.

Intravenous immunoglobulin was safe in all patients and did not cause any adverse effects.

A Annals of the Rheumatic Diseases 2003;62:1221-1223. 